\title{
A SIMPLE PROOF OF THE THEOREM OF P. J. COHEN
}

\author{
BY TAKASHI ITÔ AND ICHIRO AMEMIYA
}

Communicated by W. Rudin, June 11, 1964

Cohen, in [1], completing a line of investigations initiated by Helson [2] and Rudin [4], proved the theorem which determines the form of idempotent measures on locally compact abelian groups. As his original proof as well as its version by Rudin [5] is rather complicated, we will give, in this paper, a simple proof of the theorem in the form below; here a bounded measure $\mu$ on a locally compact abelian group $G$ is said to be canonical if $\mu$ satisfies the following conditions: The Fourier transform $\hat{\mu}$ of $\mu$ is an integral valued function and $\mu$ is absolutely continuous with respect to the Haar measure of a certain compact subgroup of $G$, namely $\mu=\sum_{i=1}^{n} n_{i} \gamma_{i} \cdot m$ where $n_{i}$ is integer, $\gamma_{i}$ a continuous character, and $m$ the Haar measure of a compact subgroup.

THEOREM. Every bounded measure on $G$ with integral valued Fourier transform is a sum of a finite number of mutually orthogonal canonical measures.

As Rudin showed in [4], it is sufficient to prove the theorem for compact $G$. So, hereafter, let $G$ be a compact abelian group.

Our proof is based on the following facts, (i) and (ii). We consider a set $A$ of measures of the form $\gamma \cdot \mu$ where $\gamma$ ranges over a set of continuous characters on $G$ and $\mu$ is a fixed bounded measure on $G$. Let $\nu$ be an accumulation point of $A$ in the weak ${ }^{*}$-topology ${ }^{1}$ on the space $M(G)$ of bounded measures on $G$, then we have:

(i) For any compact subgroup $G_{0}$ of $G, \nu$, inside $G_{0}$, either coincides with some $\gamma \cdot \mu$ in $A$ or is singular with respect to the Haar measure of $G_{0}$;

(ii) $\|\nu\|<\|\mu\|$, if $\hat{\mu}$ is integral valued and $\mu \neq 0$.

(i) can be proved as follows: The restriction of $\nu$ to $G_{0}$ either coincides with a restriction of some $\gamma \cdot \mu$ in $A$ to $G_{0}$ or is also an accumulation point of the restrictions of the $\gamma \cdot \mu$ to $G_{0}$ in the weak ${ }^{*}$-topology of $M\left(G_{0}\right)$. In the latter case, applying Helson's lemma ${ }^{2}$ to the group $G_{0}$, the restriction of $\nu$ to $G_{0}$ is singular with respect to the Haar measure of $G_{0}$.

1 The weak topology on $M(G)$ as dual of $C(G)$.

${ }^{2}$ Helson's lemma [3] is an immediate consequence of the fact that the Fourier transforms of summable functions on a compact group vanish at infinity, cf. Rudin [5, Lemma 3.5.1, p. 66]. 
To prove (ii), take an arbitrary positive number $\kappa<\|\nu\| /\|\mu\|$; then we can find $f \in C(G)$ such that $\|f\|_{\infty} \leqq 1$ and

$$
\int_{G} f d \nu>\kappa\|\mu\| \text {. }
$$

Since the set of all measures $\sigma$ satisfying $\Omega \int_{G} f d \sigma>\kappa\|\mu\|$ is open and $\nu$ is an accumulation point of $\gamma \cdot \mu$ 's, there exist $\gamma_{1}, \gamma_{2}$ such that $\gamma_{1} \cdot \mu \neq \gamma_{2} \cdot \mu$ and the real part of $\int_{G} f \gamma_{1} d \mu$ and $\int_{G} f \gamma_{2} d \mu$ are both greater than $\kappa\|\mu\|$. Take $\theta$ so that $d \mu=\theta d|\mu|$, write $f \gamma_{j} \theta=g_{j}+i h_{j}$ $(j=1,2)$. Then

$$
\int_{G} g_{j} d|\mu|>\kappa\|\mu\|
$$

and hence (cf. Cohen [1, p. 206])

$$
\int_{a}\left|h_{j}\right| d|\mu|=\mathfrak{g}\left\{\int_{G}\left(g_{j}+i\left|h_{j}\right|\right) d|\mu|\right\} \leqq \sqrt{ }\left(\|\mu\|^{2}-\kappa^{2}\|\mu\|^{2}\right),
$$

so that

$$
\begin{aligned}
\int_{G}\left|1-f \gamma_{j} \theta\right| d|\mu| & \leqq \int_{G}\left(1-g_{j}\right) d|\mu|+\int_{G}\left|h_{j}\right| d|\mu| \\
& \leqq(1-\kappa)\|\mu\|+\sqrt{ }\left(1-\kappa^{2}\right)\|\mu\|,
\end{aligned}
$$

and so

$$
\int_{G}\left|\gamma_{j}-f \gamma_{1} \gamma_{2} \theta\right| d|\mu| \leqq\left(1-\kappa+\sqrt{ }\left(1-\kappa^{2}\right)\right)\|\mu\| .
$$

Hence it follows that

$$
\left\|\gamma_{1} \cdot \mu-\gamma_{2} \cdot \mu\right\|=\int_{G}\left|\gamma_{1}-\gamma_{2}\right| d|\mu| \leqq 2\left(1-\kappa+\sqrt{ }\left(1-\kappa^{2}\right)\right)\|\mu\| .
$$

On the other hand, since the Fourier transform of $\gamma_{1} \cdot \mu-\gamma_{2} \cdot \mu$ is integral valued, we have $\left\|\gamma_{1} \cdot \mu-\gamma_{2} \cdot \mu\right\| \geqq 1$ and hence we can derive an inequality

$$
\kappa<1-\frac{1}{16\|\mu\|^{2}}
$$

this proves a strengthened form of (ii), namely,

$$
\|\nu\| \leqq\|\mu\|-\frac{1}{16\|\mu\|} .
$$


THE PROOF OF THE THEOREM. Let $\mu$ be a bounded measure with integral valued Fourier transform and $A$ the set of those $\gamma \cdot \mu$ for which $\int_{G} \gamma d \mu \neq 0$. The closure $\bar{A}$ of $A$ in the weak*-topology is a compact set and does not contain 0 , since all $\int_{G \gamma} d \mu$ are integers different from 0 . Since the norm is a lower semicontinuous function in the weak*-topology, the norms of the elements in $\bar{A}$ attain the minimum value, say, at $\nu \neq 0$. If $\int_{\sigma} \gamma d \nu \neq 0$, then $\gamma \cdot \nu$ lies in $\bar{A}$, so the set of all $\gamma \cdot \nu$ with $\int_{G} \gamma d \nu \neq 0$ can not have any accumulation point, since such a point, if it exists, must be in $\bar{A}$ and according to (ii), must have norm less than $\|\nu\|$. So this set of $\gamma \cdot \nu$ is finite and we can see easily that such a measure $\nu$ vanishes outside some compact group $G_{0}$ and is absolutely continuous with respect to the Haar measure of $G_{0}$, in other words, $\nu$ is canonical.

If $\nu$ is not an accumulation point of $A$, then $\nu$ coincides with some $\gamma \cdot \mu$ and hence $\mu$ itself is canonical. If $\nu$ is an accumulation point of $A$, then, by (i), $\nu$, being not singular with respect to the Haar measure of $G_{0}$, must coincide with some $\gamma \cdot \mu$ inside $G_{0}$. In the latter case, the restriction of this $\gamma \cdot \mu$ to $G_{0}$ is canonical and hence $\mu$ has the same property. So we have a canonical measure $\mu_{1}=\chi_{G_{0}} \cdot \mu^{3}$ and an orthogonal decomposition

$$
\mu=\mu_{1}+\left(\mu-\mu_{1}\right) .
$$

The same argument is applicable to $\mu-\mu_{1}$ and, since its norm decreases at least 1 from that of $\mu$, we can attain finally the desired decomposition.

\section{REFERENCES}

1. P. J. Cohen, On a conjecture of Littlewood and idempotent measures, Amer. J. Math. 82 (1960), 191-212. 691.

2. H. Helson, Note on harmonic functions, Proc. Amer. Math. Soc. 4 (1953), 686-

3. - On a theorem of Szegö, Proc. Amer. Math. Soc. 6 (1955), 235-242.

4. W. Rudin, Idempotent measures on abelian groups, Pacific J. Math. 9 (1959), 195-209.

5. - Fourier analysis on groups, Interscience, New York, 1962.

HoKkaIDo UnIVERSITY, JAPAN

${ }^{3} \chi G_{0}$ denotes the characteristic function of the set $G_{0}$. 